Ueber die basischen Stoffe des Zellkerns ${ }^{1}$ ).

Von

A. Kossel.

Unter den Spaltungsproducten der Eiweisskörper und der Albuminoide finden wir einzelne, die nur gewissen Gruppen. von Eiweisskörpern eigen sind, andere, welche man bisher bei allen Eiweisssubstanzen aufgefunden hat. $\mathrm{Zu}$ den ersteren gehören z. B. das Glycocoll, das Tyrosin und die schwefelhaltige Gruppe, zu den letzteren Leucin und die basischen Spaltungsproducte, deren Kenntniss durch die Untersuchungen von D rech sel angebahnt worden ist. Wir müssen also annehmen, dass in allen Eiweissstoffen ein Kern vorhanden ist, welcher die basischen Spaltungsproducte und Leucin liefert.

Die Aufklärung der Constitution dieses Kerns ist natürlich von der grössten Bedeutung, und desshalb wird das Studium derjenigen eiweissartigen Substanzen, welche diesen Kern in möglichster Reinheit enthalten, die wichtigste Vorbedingung für die Aufklärung der Constitution der Eiweisskörper überhaupt bilden müssen. Die folgenden Untersuchungen zeigen, dass die Protamine derartige Substanzen sind und zwar ergiebt sich, dass diese Körper neben den basischen Spaltungsproducten nur sehr wenig oder gar keine Monoamidosäuren liefern können.

Ich nehme an, dass in sämmtlichen Eiweisskörpern der Atomcomplex des Protamins vorhanden ist. Dieser ist nicht nur der Ursprung der basischen Spaltungsproducte des Eiweisses,

1) Der Inhalt dieser Abhandlung ist bereits in den Sitzungsberichten der Kőniglich Preuss. Akademie der Wissenschaften vom 9. April 1896 mitgetheilt worden. 
sondern er bewirkt auch die allen Eiweisskörpern gemeinsame Violettfärbung mit Natronlauge und Kupfersulfat.

Das Protamin ist im Jahre 1874 von Miescher ${ }^{1}$ ) als Bestandtheil der Spermatozoen des Rheinlachses beschrieben worden. Miescher gewann diese Base durch Extraction des entfetteten Spermas mit verdünnter Salzşäure und Fällung des theilweise neutralisirten salzsauren Extractes mit Platinchlorid. Hierbei wird das Protamin als Platinverbindung gefällt, mit Schwefelivasserstoff zersetzt und durch nochmalige Fällung mit Platinchlorid gereinigt. Aus dem Platindoppelsalz lässt sich das Chlorhydrat durch Zersetzung mit Schwefelwasserstoff darstellen. Auch das Quecksilbernitrat kann als Fällungsmittel des Protamins bei der Darstellung aus den Lachssperma verwandt werden. Miescher beschreibt das. Protamin als eine starke Base, welche durch Phosphormolybdänsäure, Jodquecksylberjodkalium, Ferrocyankalium, Quecksilberchlorid, Silbernitrat und Goldchlorid aus der wăsserigen Lösung der Salze gefällt wird. Ammon ruft bei Gegenwart von Natriumsulfat oder Phosphat eine milchige Trübung hervor. Miescher analysirte das Platindoppelsalz und erhielt Zahlen, welche ihn zur Annahme der Formel $\mathrm{C}_{9} \mathrm{H}_{91} \mathrm{~N}_{8} \mathrm{O}_{3}$ veranlassten.

Die Untersuchungen Miescher's wurden in dem gleichen Jahre von Piccard ${ }^{2}$ ) fortgesetzt. Dieser Forscher analysirte ein in gleicher Weise dargestelltes Platindoppelsalz des Protamins und gelangte, zu der Formel $4 \mathrm{PtCl}_{4}+2\left(\mathrm{HCl} . \mathrm{C}_{8} \mathrm{H}_{16} \mathrm{~N}_{4^{1 / 2}} \mathrm{O}_{2}\right)$ » oder $\mathrm{C}_{16} \mathrm{H}_{38} \mathrm{~N}_{9} \mathrm{O}_{6}, 2 \mathrm{HCl}, \mathrm{PtCl}_{6}$. Piccard zeigte zugleich, dass das von Miescher dargestellte Präparat mit Guanin und Hypoxanthin verunreinigt war und dass man diese Basen aus dem Lachssperma in grösseren Mengen darstellen kann. Die Entstehung dieser Stoffe aus den Nucleïnsubstanzen wurde damals noch von Niemanden vermuthet und erst später durch

. 1) Verhandlungen der naturforschenden Gesellschaft in Basel. VI, Heft I, S. 138-208, 1874.

2) Piccard: Ueber Protamin, Guanin und Sarkin, als Bestandtheile des Lachsspermas. Berichte d. deutschen chemischen Gesellschaft (1874), Bd. 7, S. 1714. 
meine Untersuchungen über das Nucleïn der Hefe bekannt gemacht.

Nach den Arbeiten Miescher's und Piccard's ruhte die Frage nach der chemischen Natur des Protamins ungefähr 20 Jahre. Meine Untersuchungen führten mich zu der im Jahre 1894 mitgetheilten $^{1}$ ) Beobachtung, dass das Protamin mit Eiweiss oder Albumose eine Verbindung bildet, welche alle Reactionen des Histons besitzt, insbesondere auch durch Ammoniak auch ihren Lösungen gefällt wird. Ich bemühte mich, eine grössere Menge von Protamin aus Lachssperma darzustellen und es gelang mir, eine bequemere Darstellungsweise auszuarbeiten. Da indess die Beschaffung des Lachsspermas mit bedeutenden Schwierigkeiten verknüpft war, so habe ich mich bemüht, ein anderes Ausgangs-Material ausfindig zu machen und dies in dem Störsperma gefunden. Die Störtestikeln wurden mir zum grossen Theil von Herrn Pfarrer Radtke in Bohnsack bei Danzig, Beisitzer in Vorstande des westpreussischen Fischerei-Vereins, verschafft, wofür ich demselben auch an dieser Stelle meinen besten Dank abstatte.

Darstellung des schwefelsauren Protamins aus den Testikeln von Lachs und Stör.

Die reifen oder der Reife sehr nahen Testikeln werden zerhackt, die zerhackte Masse mit Wasser anhaltend geschüttelt, durch ein Tuch geseiht und die milchige colirte Flüssigkeit mit einigen Tropfen Essigsäure versetzt. Hierdurch bewirkt man, dass die im Wasser suspendirten morphotischen Elemente sich zusammenballen. Die Flüssigkeit wird filtrirt, der Filterrückstand mehrmals mit Alkohol ausgekocht, sodann mit Aether extrahirt und bei Zimmertemperatur getrocknet.

100 gr. dieser Spermamasse werden mit $500 \mathrm{cbcm}$. einprocentiger Schwefelsäure 1/\% Stunde lang im Schüttelcylinder behandelt, auf der Nutsche abgesaugt und diese Extraction noch dreimal mit der gleichen Menge Schwefelsäure wiederholt. Das Filtrat wird mit der dreifachen Menge Alkohol gefällt,

1) Ueber die Lymphzellen, Deutsche medicinische Wochenschrift. 1894, Nr. 7 . 
die Flüssigkeit nach 12 bis 24 Stunden decantirt und der Niederschlag, welcher aus schwefelsaurem Protamin besteht, abgesaugt. Die Ausbeute an diesem Rohproduct beträgt etwa $20 \%$ der trockenen Spermamasse. Zur weiteren Reinigung kann man das Sulfat nochmals in Wasser lösen und die Lösung nach Neutralisation durch Ammoniak mit Alkohol fällen. Durch mehrfache Wiederholung dieser Manipulation erhält man ein rein weisses Salz, welches sich in Wasser zu einer farblosen Flüssigkeit auflöst.

Bei dem Protamin des Lachsspermas, dessen Analyse unten mitgetheilt ist, wurde noch folgende Reinigungsmethode angewandt. Die Lösung des Sulfats wurde mit wässeriger Lösung von Natriumpikrat gefällt. Das Pikrat, welches sich sofort abscheidet, wurde abgesaugt, ausgewaschen, in Wasser zertheilt, mit einem Ueberschuss von Schwefelsäure versetzt. und durch Ausschütteln mit Aether von der Pikrinsäure befreit. Aus der schwefelsauren Lösung des Protamins wurde das Sulfat durch Fällung mit Alkohol gewonnen.

Figenschaften und Zusammensetzung der Protamine.

Das aus Lachssperma dargestellte Sulfat wurde in Wintersemester 1894/95 auf meine Veranlassung von Herrn Alexander Wolkowicz analysirt und ergab folgende Zahlen:

\begin{tabular}{|c|c|c|c|c|c|c|}
\hline & \multicolumn{5}{|c|}{ Gefunden: } & \multirow{2}{*}{$\begin{array}{c}\text { Berechnet für } \\
\mathrm{G}_{16} \mathrm{H}_{81} \mathrm{~N}_{8} \ddot{\mathrm{O}}_{8}, \mathrm{H}_{2} \mathrm{SO}_{4}=\end{array}$} \\
\hline & I. & II. & III. & IV. & v. & \\
\hline & 38,67 & $\mathbf{3 8 , 3 5}$ & 38.40 & - & - & 38,79 \\
\hline & 6,97 & 6,86 & 6,96 & - & - & 6,67 \\
\hline & - & - & - & 25,20 & - & 25,45 \\
\hline${ }_{2} \mathrm{SO}_{4}$ & - & - & - & - & 19.59 & 19,79 \\
\hline
\end{tabular}

Diese Ergebnisse lassen die Formel $\mathrm{C}_{16} \mathrm{H}_{31} \mathrm{~N}_{8} \mathrm{O}_{3}, \mathrm{H}_{2} \mathrm{SO}_{4}$ als die wahrscheinlichste erscheinen. Gleichzeitig mit der vorläufigen Mittheilung dieser Formel in den Sitzungsberichten der Kgl. Preuss. Akademie der Wissenschaften vom 9. April d. J. ist eine Publication Schmied deberg 's erschienen ${ }^{1}$ ), in welcher aus der Analyse des Platinsalzes die Formel $\mathrm{C}_{16} \mathrm{H}_{98} \mathrm{~N}_{9} \mathrm{O}$, für das Protamin des Lachsspermas abgeleitet wird. Diese Formel ist unmöglich, da sie der bekannten Anforderung, dass die

1) Archiv f. exp. Pathologie und Pharmakologie, Bd. 37, S. 100-155. 
Summe der Valenzen durch 2 dividirbar sein muss, nicht entspricht. Die um ein Atom Wasserstoff reichere Formel $\mathrm{C}_{16} \mathrm{H}_{99} \mathrm{~N}_{9} \mathrm{O}$, würde sich von der meinigen durch den Mindergehalt von einem Molekül Wasser unterscheiden. Ob etwa das Sulfat bei stärkerem Trocknen noch Wasser verliert. habe ich nicht untersucht.

Bei der Verarbeitung des Störspermas nach der eben angegebenen Methode gewann ich eine Substanz, welche in ihren Eigenschaften eine sehr grosse Aehnlichkeit mit dem Protamin des Lachsspermas zeigte, aber hinsichtlich ihrer Zusammensetzung und einzelner Reactionen von dem Lachsprotamin abweicht.

In Anbetracht der nahen chemischen und physiologischen . Beziehungen wird man für beide Stoffe einen gemeinsamen Gruppennamen wählen müssen, und hiezu ist die von Mies cher eingeführte Bezeichnung "Protamin » am besten geeignet. An Stelle der schleppenden Namen «Lachsprotamin » und «Störprotamin » schlage ich die Worte «Salmin» und «Sturin » vor.

Die Untersuchungen über die Zusammensetzung des Sturins sind noch nicht zu einem befriedigenden Abschluss gelangt und ich werde die Resultate der Analysen später mittheilen.

Das Sulfat des Salmins ist in heissem Wasser leicht löslich und scheidet sich aus einer nicht zu verdünnten Lösung beim Erkalten als Oel ab. In einem geringen Ueberschuss der Säure löst es sich auch in der Kälte leicht auf. Das Sturinsulfat bleibt beim Erkalten selbst einer 10 procentigen Lösung gelöst. Die wässerige Lösung der Sulfate wird durch die Salze schwerer Metalle, z. B. Silbernitrat, Quecksilberchlorid, ferner durch Phosphorwolframsäure; Jodwismuthjodkalium, Jodjodkalium, Ferrocyankalium gefällt, wie dies z. Theil bereits von Miescher angegeben ist. Natriumpikrat erzeugt den unlöslichen Niederschlag von Salmin- und Sturinpikrat. Ammoniak erzeugt in einer nicht zu verdünnten Lösung. des Sturinsulfats eine ölige Fällung, Natron im Ueberschuss löst die Base.

Sehr bemerkenswerth ist das Verhalten dieser Körper zu Kochsalz. Verșetzt man eine Lösung von Salminsulfat, 
welche einen geringen Säureüberschuss enthält, mịt dem gleichen Volumen einer gesättigten Kochsalzlösung, so bildet sich eine milchige Trübung und nach einigen Stundén ist ein sehr beträchtlicher Theil des Salminsalzes am Boden des Gefässes angesammelt. Durch Zusatz von mehr Kochsalzlösung kann man aus einer nicht $z u$ verdünnten Lösung fast die ganze Menge des Salmins abscheiden. Während eine 5 procentige saure Lösung von Salminsulfat durch das gleiche Volumen gesättigter Kochsalz gefällt wird, bleibt selbst eine 10 procentige Lösung des Sturinsulfats bei gleicher Behandlung klar, erst Zusatz von mehr Kochsalz ruft Fällung hervor.

Dies Verhalten kann natürlich mit Vortheil bei der Darstellung des Salninchlorbydrates benutzt werden, indem man das salzsaure Extract des entfetteten Lachsspermas mit dem 2-3 fachen Volumen Kochsalzlösung fällt, das Oel nach 1-2 Tagen abtrennt und durch Wiederholung dieser Fällung und zuletzt durch Abscheidung mit Alkohol reinigt.

Sowohl das Salmin wie das Sturin halten Kupfer mit violetter Farbe in alkalischer Lösung, sie geben also die «Biuretreaction "schon bei gewöhnlicher Temperatur. Die Mill onsche Reaction bleibt - wie schon Miescher für das Salmin angegeben hat - aus.

Fügt man zu einer alkalischen Lösung der Protamine Benzoylchlorid, so fallen die schwer löslichen Benzoylverbindungen aus. Bei dem Versuche zur Feststellung der Molekulargrösse des Salmins ergab sich, dass $0,7126 \mathrm{gr}$. der freien Base keine Erhöhung des Siedepunktes von Wasser hervorriefen.

Protaminähnliche Stoffe konnte ich auch in den Testikeln anderer Salmoniden z. B. der Bachforelle und des Schnäpels (Coregonus oxyrhynchus) nachweisen.

\section{Die Spaltungsproducte des Sturins.}

Die Spaltung des Sturins wurde in folgender Weise ausgeführt :

$20 \mathrm{gr}$. Sturinsulfat werden mit einer Mischung von $60 \mathrm{gr}$. conc. Schwefelsäure und $120 \mathrm{cbcm}$. Wasser 8 Stunden am 
stallisirender Syrup erhalten; derselbe färbte sich beim Kochen mit alkalischer Bleizuckerlösung braun - enthielt also etwas locker gebundenes Schwefel. Die schwefelhaltige Verbindung habe ich in krystallisirtem Zustande nicht erhalten können; indessen liess sich dieselbe durch vorsichtigen Zusatz von Bleiessig und Ammoniak aus der Lösung entfernen. Das Filtrat vom Bleiniederschlag wurde mit Schwefelwasserstoff von Blei befreit, gekocht und mit Silbernitrat versetzt. Dabei blieb die Lösung klar, aber bei vorsichtigem Zusatz von Ammoniak entstand ein voluminöser Niederschlag. Ammoniak darf nur so lange zugegeben werden, als noch eine Fällung entsteht, weil dieselbe sich in überschüssigem Ammoniak leicht auflöst. Der Niederschlag wurde abfiltrirt, mit ein wenig Salzsäure zerlegt, die Lösung von Chlorsilber filtrirt und auf dem Wasserbade eingetrocknet. Da der Silberniederschlag immer ein wenig essigsaures Silberoxyd enthält, geht beim Eintrocknen die Essigsäure mit der überschüssigen Salzsäure fort. Der dunkelbraune Rückstand wurde mit Thierkohle entfärbt und in stark concentrirter Lösung zum Krystallisiren hingestellt. Nach einiger Zeit schieden sich schöne, tafelförmige Krystalle aus, welche nach Umkrystallisiren über Schwefelsāure getrocknet und analysirt wurden.

1. 0,2256 gr. gab 0,2878 gr. $\mathrm{CO}_{2}=0,0785$ gr. C. und 0,1158 gr. $\mathrm{H}_{2} \mathrm{O}$ $=0,0129 \mathrm{gr}$. $\mathrm{H}$.

2. 0,1701 gr. gab 0,2153 gr. $\mathrm{CO}_{2}=0,0587$ gr. $\mathrm{C}$ und 0,0858 gr. $\mathrm{H}_{2} \mathrm{O}$ $=0,0095$ gr. $\mathrm{H}$ :

3. 0,2065 gr. gab nach Kjeldahl $\mathrm{NH}_{8}$, das durch $29,7 \mathrm{cbcm}$. ${ }^{1} / 10$ Normalsäure neutralisirt wurde, $=0,04158 \mathrm{gr}$. $\mathrm{N}$.

4. $0,1503 \mathrm{gr}$ : gab $0,1024 \mathrm{gr}$. $\mathrm{Ag} \mathrm{Cl}=0,0253 \mathrm{gr}$. $\mathrm{Cl}$.

Hieraus ergibt sich die Formel $\mathrm{C}_{6} \mathrm{H}_{12} \mathrm{~N}_{3} \mathrm{O}_{3} \mathrm{Cl}$.

\begin{tabular}{|c|c|c|c|c|c|c|}
\hline & & & & f.: & & \\
\hline & Ber.: & 1. & 2. & 3. & 4. & \\
\hline $72^{\circ}$ & 34,37 & 34,80 & 34,52 & - & -0 & $\%$ \\
\hline $\mathrm{H}_{12} 12$ & 5,72 & 5,70 & 5,60 & - & - & $\gg$ \\
\hline $\mathrm{N}_{3} \quad 42$ & 20,05 & - & - & 20,14 & - & 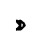 \\
\hline 35,5 & 16.94 & - & - & - & 16,85 & 5 \\
\hline $\mathrm{O}_{3} \quad 48$ & - & $\therefore$ & - & - & - & , \\
\hline 209,5 & & & & & & \\
\hline
\end{tabular}


Das Salz enthält : 1 Mol. Krystallwassèr, das : während stundenlangen Erhitzens bei $120^{\circ}$ nicht entweicht, aber bei $135^{\circ}$ unter schwacher Braunfärbung des Salzes fortgeht.

0,256 gr. verloren bei $135 \% 0,023 \mathrm{gr}$. $=8,98 \%$. Berechnet wird für $\mathrm{C}_{6} \mathrm{H}_{10} \mathrm{~N}_{8} \mathrm{O}_{2} \mathrm{Cl}+\mathrm{H}_{2} \mathrm{O} 8,59 \%$.

Die Ausbeute war eine sehr geringe: aus der in Arbeit genommen Eiweissmenge (1125 gr.) wurde nur 3-4 gr. reines Chlorhydrat erhalten.

In derselben Weise wurde das nämliche Chlorid aus eingetrocknetem Blutserum dargestellt:

0,2203 gr. gaben $0,2763 \mathrm{gr} . \mathrm{CO}_{2}=0,07535 \mathrm{gr}$. $\mathrm{C}$ und $0,1092 \mathrm{gr} . \mathrm{H}_{2} \mathrm{O}$ $=0,01214 \mathrm{gr}$. H.

$$
\begin{aligned}
& \text { Ber. für }
\end{aligned}
$$

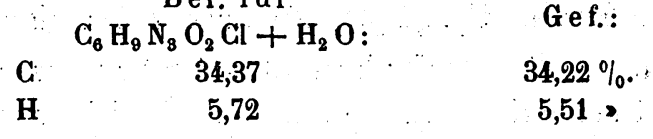

Aus Hornsubstanz dargestellt ergab die Substanz folgende Analysenwerthe:

0,145 gr. gaben 0,1835 gr. $\mathrm{CO}_{2}=0,05$ gr. $\mathrm{C}$ und $0,0714 \mathrm{gr}$. $\mathrm{H}_{2} \mathrm{O}$ $0,00793 \mathrm{gr}$. H.

$\begin{array}{ccc} & \text { Ber.: } & \\ \text { C } & 34,37 & \text { Gef.: } \\ \text { H } & 5,72\end{array} \quad \begin{gathered}34,50 \% \\ \text { G,47 }\end{gathered}$

Dàs Ghlorhydrat ist optisch inactiv. In Wasser ist es ziemlich leicht löslich aber unlöslich in Alkohol, sowie in Aether. Die wässerige Lösung reagirt stark sauer.

Aứs dem Chlorhydrat wurde die freie Base dadurch erhalten, dass dasselbe mit einer äquivalenten Menge Silbersulfat in Sulfat übergeführt wurde, wonach dieses Salz mit Barythydrat zerlegt und der Barytüberșchuss mit Kohlensäure entfernt wurde. Die Lösung setzt beim Concentriren nadelförmige oder táfelförmige Krystalle $a b$. Dieselben wurden über Schwefelsautre getrocknet und analysirt.

1. 0,1913 gr. gaben 0,3269 gr. $\mathrm{G}=0,08915 \mathrm{gr}$. $\mathrm{C}$ und $0,0967 \mathrm{gr} . \mathrm{H}_{2} \mathrm{O}$ $=0,01074 \mathrm{gr}$. $\mathrm{H}$.

2. 0,1545 gr. gaben $\mathrm{NH}_{3}$, das durch $29,8 \mathrm{cbcm}, 1 / 10$ Normalsäure neutralisirt wurde $=0,0417 \mathrm{gr}$. $\mathrm{N}$. 
Die Analyse des Histidins ergab folgende Zahlen:

\begin{tabular}{|c|c|c|c|c|c|c|}
\hline & & Gefu & nden & & Bere & $\mathrm{für}$ \\
\hline & I. & II. & III. & IV. & $\mathrm{C}_{12} \mathrm{H}_{20} \mathrm{~N}_{6} \mathrm{O}_{4}$ & $\mathrm{C}_{6} \mathrm{H}_{9} \mathrm{~N}_{3} \mathrm{O}_{2}$ \\
\hline C & 46,07 & 46,16 & $.46,56$ & - & 46,15 & 46,45 \\
\hline $\mathrm{H}$ & 6,56 & 6,24 & 6,07 & - & 6,41 & 5,81 \\
\hline $\mathbf{N}$ & - & - & - & 27,30 & 26,92 & 27,10 \\
\hline
\end{tabular}

Die freie Base ist in Wasser zu einer alkalischen Flüssigkeit löslich, sehr wenig löslich in Alkohol und unlöslich in Aether.

Nach den vorliegenden Analysen kann man noch nicht entscheiden, ob dem Histidin die Formel $\mathrm{C}_{12} \mathrm{H}_{20} \mathrm{~N}_{8} \mathrm{O}_{4}$. oder $\mathrm{C}_{6} \mathrm{H}_{9} \mathrm{~N}_{8} \mathrm{O}_{2}$ zukommt. Die Analysen II und III der freien Base stimmen besser zu der Formel $\mathrm{C}_{6} \mathrm{H}_{9} \mathrm{~N}_{8} \mathrm{O}_{2}$ ). während eine Molekulargewichtsbestimmung durch Erhöhung des Siedepunktes von Phenol ein Molekulargewicht von 296 ergab, welches hinreichend mit der von der Formel $\mathrm{C}_{18} \mathrm{H}_{90} \mathrm{~N}_{6} \mathrm{O}_{4}$ geforderten Zahl 312 übereinstimmt. Indess ist dieser Versuch nicht ausschlaggebend und ich muss die Entscheidung die $n$ Frage weiteren Untersuchungen überlassen, welche ich nach Beschaffung von mehr Material anzustellen gedenke.

Die übrigen Spaltungsproducte.

Die vom Quecksilberchlorid-Niederschlag abfiltrirte Flüssigkeit wurde durch Schwefelwasserstoff vom Quecksilber befreit, mit Sehwefelsäure versetzt und mit. Phosphorwolframsäure in der Wärme gefällt. Der Niederschlag - verwandelt sich bald in feine Krystallnadeln. Der durch Absaugen erhaltene Niederschlag wird in viel heissem Wasser gelöst, zur Entfernung der Phosphorwolframsäure mit überschüssiger Barytlösung versetzt und der Ueberschuss des. Baryts durch Einleiten von Kohlensäure entfernt. Beim Eindampfen der Flüssigkeit scheiden sich Krystalle eines kohlensauren Salzes aus, welche bei öfterem Umkrystallisiren aus Wasser die Kohlensäure bis auf Spuren verlieren. Die Analyse zeigte, dass in ihnen das von Schulze und Steiger ${ }^{1}$ ) entdeckte Arginin vorlag.

1) Diese Zeitschrift, Bd. 11, S. 49 . 


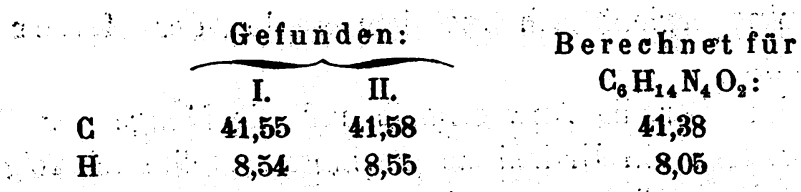

Das Arginin kann sehr leicht durch das von Hedin') kürzlich beschriebene schwer lösliche, gut krystallisirende basische Silbersalz characterisirt werden. Ich führte daher das Carbonat nach den Angaben des genannten Forschers in dieses Salz über und erhielt bei der Analyse folgende Zahlen:

$\begin{array}{ccc} & \text { Gefunden: } & \text { Berechnet für } \\ & \mathrm{C}_{6} \mathrm{H}_{14} \mathrm{~N}_{4} \mathrm{O}_{2}+\mathrm{AgNO}_{8}+\mathrm{H}_{2} \mathrm{H}_{2} \mathrm{O}: \\ \mathrm{C} & 20,78 & 20,40 \\ \mathrm{H} & 4,79 & 4,25 \\ \mathrm{~N} & 20,12 \text { (vol.) } & 19,83\end{array}$

Ebenso erhielt ich auch das zweite von Hedin beschriebene Silbersalz $\mathrm{C}_{6} \mathrm{H}_{14} \mathrm{~N}_{4} \mathrm{O}_{2}, \mathrm{HNO}_{8}+\mathrm{Ag} \mathrm{NO}, \mathrm{H}_{3}$ welches in Nadeln krystallisirt. Dasselbe ergab bei der Analyse:

$4 ?$

$$
\begin{array}{ccc}
\text { Gefunden: } & \begin{array}{c}
\text { Berechnet für } \\
\mathrm{Ag}
\end{array} \mathrm{C}_{6} \mathrm{H}_{14} \mathrm{~N}_{4} \mathrm{O}_{2}, \mathrm{HNO}_{3}+\mathrm{AgNO}_{8}: \\
26,58
\end{array}
$$

Somit ist die Entstehung des Arginins bei der hydrolytischen Spaltung des Protamins völlig sicher gestellt. Hedin ${ }^{2}$ ) hat das Arginin auch unter den Zersetzungsproducten der Eiweisskörper aufgefunden und hat es wahrscheinlich gemacht, dass das Lysatin Drechsel's als eine Mischung von gleichen Molekülen Arginin und Lysin aufzufassen ist.

Das Histidin und das Arginin sind nicht die einzigen Spaltungsproducte des Sturins, stets bleibt eine nicht unbeträchtliche Menge stickstoffhaltiger organischer Substanz von basischen Eigenschaften in der Mutterlauge zurück.

Von besonderem Interesse erschien mir die Frage, ob. die Protamine bei ihrer Spaltung auch Ámidosäuren liefern. Die Abwesenheit der tyrosinbildenden Gruppe im Molekül des

1) Diese Zeitschrift. Bd. 21, S. 297.

2) Diese Zeitschrift, Bd. 20, S 186 und Bd. 21, S. 155. 
Protamins ergiebt sich aus dem Fehlen der Millon'schen Reaction.

Um über die Anwesenheit und die Menge der übrigen Amidosäuren ein Urtheil zu gewinnen, stellte ich folgenden Versuch an. Eine genau abgewogene Menge Protaminsulfat, dessen Stickstoffgehalt durch Kjeldahl's Methode festgestellt war, wurde 8 Stunden in dem oben angegebenen Verhältniss mit Schwefelsäure am Rückflusskühler gekocht, die Reactionsmasse auf $50 \mathrm{cbcm}$. verdünnt, mit Phosphorwolframsäure gefält, der Niederschlag abfiltrirt und das Filtrat nach $\mathrm{Kj} \mathrm{el-}$ dahl auf seinen Stickstoffgehalt untersucht. Da die basischen Stoffe durch Phosphorwolframsäure fällbar sind, die Monoamidosāuren hingegen nicht, so kann der Stickstoffgehalt dieses Filtrats als Ausdruck für die Menge der Monoamido-säuren betrachtet werden. Es ergab sich nun das. Verhältniss des Gesammtstickstoffs zu dem durch Phosphorwolframsäure nicht fâllbaren Stickstoff im ersten Versuch wie $100: 6,7$, im zweiten Versuch wie $100: 6,4$. Wenn also überhaupt Amidosäuren bei der Spaltung des Protamins entstehen, so kann ihre Menge nur äusserst gering sein.

Das Sturin unterscheidèt sich also In seiner Constitution von den Peptonen dadurch, dass ihm diejenige Gruppe, aus welcher die Amidosäuren hervorgehen, fehlt oder dass sie wenigstens an Menge sehr bedeutend zurücktritt.

Ferner ergibt sich, dass die Biuretreaction, wie schon oben erwähnt, an denjenigen Atomcomplex gebunden ist, welcher die basischen Spaltungsproducte des Eiweisses liefert.

Wie ich schon früher mitgetheilt habe ${ }^{1}$ ), sind die Protamine im Stande, sich mit löslichen Eiweisskörpern zu vereinigen und hierbei entstehen Verbindungen, welche sich vom Hist on nicht unterscheiden lassen. Ebenso wie die Nueleînsâure bei saurer Reaction der Lösung, so vereinigt sich das Protamin bei alkalischer Reaction mit Eiweisskörpern und man erhält daher einen Niederschlag, wenn man eine ammoniakalische Lösung von Protamin zu einer ammoniakali-

1) Deutsche med. Wochenschr., 1894, Nr. 7. 
schen Eiweisslösung hinzufügt. Durch eine solche Anfügung won Protamin an Eiweiss müssen natürlich neue Eiweisssubstanzen entstehen, welche mehr Arginin oder überhaupt mehr Basen liefern. Nimmt man an, dass diese Anfügung much in der Zelle vor sich geht, so ergiebt sich eine Erklārung für die von $\mathrm{Hedin}$ gefundene Thatsache, dass die werschiedenen Eiweisskörper bei der Hydrolyse verschiedene Mengen von Arginin ergeben. 